\title{
Star Formation in the Galactic Center
}

\author{
Jens Kauffmann \\ Max-Planck-Institut für Radioastronomie, Auf dem Hügel 69, 53125 Bonn, Germany \\ email: jens.kauffmann@gmail.com
}

\begin{abstract}
Research on Galactic Center star formation is making great advances, in particular due to new data from interferometers spatially resolving molecular clouds in this environment. These new results are discussed in the context of established knowledge about the Galactic Center. Particular attention is paid to suppressed star formation in the Galactic Center and how it might result from shallow density gradients in molecular clouds.
\end{abstract}

Keywords. stars: formation, ISM: clouds, Galaxy: center

\section{Introduction}

The Galactic Center environment provides unique conditions for star formation within the Milky Way. About 3-10\% of the total molecular gas and star formation (SF) of the Milky Way $\dagger$ reside at $|\ell| \leqslant 3^{\circ}$ (i.e., within galactocentric radii $\leqslant 430 \mathrm{pc}$ ). Unlike the rest of the the Milky Way, the region within $\sim 200$ pc galactocentric radius (i.e., $|\ell| \lesssim 1.5$ ) is dominated by gas in molecular instead of atomic form. This domain of the Galaxy is therefore also known as the Central Molecular Zone (CMZ; Morris \& Serabyn 1996). The molecular clouds in the CMZ are unusually warm, dense, and "turbulent" (see below).

This text largely ignores the immediate environment of Sgr A* and the question why the CMZ is so unusual within the Milky Way. Kruijssen et al. (this volume) discuss this latter aspect of CMZ science. Instead this text sets out to summarize our knowledge about ongoing star formation and the state and distribution of dense molecular gas in the CMZ. Many of the most recent results concern the internal structure of CMZ clouds as resolved by interferometers. Many of the global aspects described in the reviews by Güsten (1989) and Morris \& Serabyn (1996) thus still remain valid today. A Galactic Center distance of $(8.34 \pm 0.16) \mathrm{kpc}$ is adopted (Reid et al. 2014).

\section{Gas and Young Stars in the Galactic Center}

\subsection{Observations of Young Stars and Star Formation}

The CMZ is a spectacular star-forming environment. It contains massive and compact young stellar groups like the Arches and Quintuplet clusters (Nagata et al. 1990, 1995; Cotera et al. 1994) that together contain $\gtrsim 200$ O-type stars (Figer 2004) and have few - but still some - counterparts in the disk of the Milky Way (Portegies Zwart et al. 2010). The CMZ also harbors the Sgr B2 molecular cloud that alone hosts 59 compact HiI regions (Gaume et al. 1995).

$\dagger$ Observations of line and dust emission indicate that the $|\ell| \leqslant 3^{\circ}$ region contains of order (3 to 8$) \times 10^{7} M_{\odot}$ of molecular gas (Dahmen et al. 1998; Tsuboi et al. 1999; Longmore et al. 2013a). It forms stars at a rate of order $0.1 M_{\odot} \mathrm{yr}^{-1}$ (Yusef-Zadeh et al. 2009; Immer et al. 2012b; Longmore et al. 2013a; Koepferl et al. 2015). The Milky Way contains $(1.0 \pm 0.3) \times 10^{9} M_{\odot}$ of molecular gas (Heyer \& Dame 2015), while the star formation rate is 1 to $4 M_{\odot} \mathrm{yr}^{-1}$ (Diehl et al. 2006; Misiriotis et al. 2006; Lee et al. 2012). 
Still, relatively few and poor overall constraints on the young stars inhabiting the CMZ exist. For example, Spitzer can at best detect embedded young stars with luminosities $\gtrsim 10^{3} L_{\odot}$ in the CMZ (Eq. [2] of Dunham et al. 2008, degraded by a factor 100 given lower completeness in the CMZ taken from Evans et al. 2007 and Gutermuth \& Heyer 2015). But some CMZ clouds have high optical depths even at wavelengths $\sim 100 \mu \mathrm{m}$ (e.g., G0.253+0.016; Lis \& Menten 1998). In these cases embedded star formation does at best manifest in subtle trends affecting the spectral energy distribution of the entire cloud (Lis et al. 2001). Similarly, OB-type stars outside clouds, which provide information on star formation during the past few $10^{6} \mathrm{yr}$, are hard to detect behind foreground extinction at the level of $A_{K_{\mathrm{s}}} \approx 2 \mathrm{mag}$ (Schödel et al. 2010; Longmore et al. 2012), corresponding to $A_{V} \approx 18 \mathrm{mag}$ (for $A_{K} / A_{V}=0.113$; Rieke \& Lebofsky 1985).

The available data provide interesting insights, though. Yusef-Zadeh et al. (2009) identify about 550 candidate young stellar objects from Spitzer images at 8 and $24 \mu \mathrm{m}$ wavelength. Immer et al. (2012b) use a similar but slightly refined technique to identify 1,141 candidate young stars in $I S O$ and $M S X$ data. These infrared observations yield star formation rates $\approx 0.1 M_{\odot} \mathrm{yr}^{-1}$ at at $|\ell| \leqslant 1.5$. The nature of these objects is, however, not entirely clear. Yusef-Zadeh et al. (2009) in particular point out that their objects have spectral energy distributions consistent with being deeply embedded in molecular clouds. But the Yusef-Zadeh et al. sources are at the same time found to typically reside away from the clouds that could envelope young stars. This produces an unclear picture. Koepferl et al. (2015) suggest that main sequence stars "illuminating" diffuse CMZ gas could produce infrared signals resembling those of embedded young stars. This complication has the potential to massively reduce the value of infrared CMZ data.

Also, Paschen $-\alpha$ imaging of the CMZ reveals an extended population of high-mass stars (Wang et al. 2010; Dong et al. 2011, 2012). Interestingly, these stars might well have formed as part of the Arches and Quintuplet clusters but then migrated to their current location (Habibi et al. 2014).

The best constraints on the total star formation activity of the CMZ might therefore come from indirect methods. For example, the number of ionizing photons produced in the CMZ can be estimated from radio data (e.g., Mezger \& Pauls 1979 for some early research). This in turn constrains the number of high-mass stars emitting such photons, which itself can be related to the star formation rate via further assumptions. Early work (e.g., Güsten 1989: 0.3 to $0.6 M_{\odot} \mathrm{yr}^{-1}$ at $|\ell| \leqslant 3.5$ ) is broadly consistent with current estimates (e.g., Longmore et al. 2013a: $\leqslant 0.06 M_{\odot} \mathrm{yr}^{-1}$ at $|\ell| \leqslant 1^{\circ}$ ). Section 4.1 describes how water and methanol masers can be used for similar but more uncertain estimates.

\subsection{State and Distribution of Dense Gas}

The distribution of molecular gas in the Galactic Center at $|\ell| \leqslant 5^{\circ}$ is highly asymmetric. About $75 \%$ of the gas seen in ${ }^{13} \mathrm{CO}$ and $\mathrm{CS}$ resides at $\ell>0^{\circ}$ and radial velocities $>0 \mathrm{kms}^{-1}$ (Bally et al. 1988). Interestingly, this distribution is opposite to potential asymmetries in star formation that are possibly seen in the aforementioned Spitzer data (Yusef-Zadeh et al. 2009). It is likely that the gas seen on these large spatial scales follows so-called $x_{1}$ orbits that are closed and elongated along the Milky Way's bar (Contopoulos \& Mertzanides 1977; Binney et al. 1991). These orbits are also dynamically stable down to some minimum size.

Provided orbits are chiefly controlled by a bar-like potential, stable trajectories on spatial scales below those of $x_{1}$ orbits are part of the family of $x_{2}$ orbits. These are closed and elongated perpendicular to the bar. Interestingly, Molinari et al. (2011) argue that the gas within $|\ell| \lesssim 1^{\circ}$ from the Galactic Center forms a system of unusually dense and massive molecular clouds with kinematics that are broadly consistent with those of 
gas on $x_{2}$ orbits. These clouds include regions like Sgr B2, G0.253+0.016, and all the other objects that form the main topic of this text.

However, Henshaw et al. (2016) recently demonstrated that the kinematics of clouds at $|\ell| \lesssim 1^{\circ}$ are much better explained by the open orbits Kruijssen et al. (2015) propose for an azimuthally symmetric CMZ potential taken from Launhardt et al. (2002). These trajectories are not stable: the orbits can form intersection points where energy can be consumed in strong shocks. The orbits are not necessarily occupied by continuous streams of gas, though, and they oscillate (and thus potentially avoid another) perpendicular to the Galactic Plane. Numerical orbit simulations like those by Lucas (2015) indicate that gas can reside on these orbits for several $10 \mathrm{Myr}$. The orbital period of major CMZ clouds like Sgr B2 is about $2 \mathrm{Myr}$ in radius and 4 Myr in azimuth (Kruijssen et al. 2015).

Binney et al. (1991) suggest that $x_{1}$ orbits shrinking below the size of stable trajectories eventually shock and dump their material onto the inner CMZ (e.g., $x_{2}$ orbits). Lucas (2015) shows that the injection of a single compact cloud into the central $\sim 100 \mathrm{pc}$ produces "streams" similar to those considered by Kruijssen et al. (2015).

CMZ molecular clouds have unusually high mean $\mathrm{H}_{2}$ densities $\sim 10^{4} \mathrm{~cm}^{-3}$ and column densities $\sim 10^{23} \mathrm{~cm}^{-2}$ (e.g., Lis \& Carlstrom 1994). The diffuse ionized gas is pervaded by a strong magnetic field $\sim 10^{3} \mu \mathrm{G}$ (Yusef-Zadeh et al. 1984; Uchida et al. 1985; Chuss et al. 2003; Novak et al. 2003) that also penetrates the CMZ clouds (Pillai et al. 2015).

CMZ molecular clouds have line widths much in excess of Galactic Disk clouds (Sec. 4.3). Many CMZ clouds appear to be subject to violent processes like cloud-cloud collisions at high velocities. This is indicated by widespread emission from SiO (Martín-Pintado et al. 1997; Hüttemeister et al. 1998; Riquelme et al. 2010b) and other molecules likely ejected from grain surfaces via shocks (Requena-Torres et al. 2006, 2008), and methanol masers excited in collisions (Mills et al. 2015; also see Menten et al. 2009, though).

Bulk gas temperatures from line ratios are typically in the range 50 to $100 \mathrm{~K}$ (Güsten et al. 1981; Ao et al. 2013; Ott et al. 2014; Ginsburg et al. 2016; also see Riquelme et al. 2010a, 2012). Hüttemeister et al. (1993) do, however, point out that the $\mathrm{NH}_{3}$-derived temperature in a given cloud varies between $\approx 25 \mathrm{~K}$ and $\gtrsim 200 \mathrm{~K}$ (also see Mills \& Morris 2013), and that the cold material contains $\sim 75 \%$ of the mass traced by $\mathrm{NH}_{3}$. This topic should be revisited systematically. It has been suggested that temperatures from some line ratios are unphysically high due to formation pumping (Lis et al. 2014), but this requires densities below the few $10^{4} \mathrm{~cm}^{-3}$ characteristic of the CMZ (D. Lis, priv. comm.). Gas temperatures $\gtrsim 50 \mathrm{~K}$ would be mysteriously decoupled from the much lower dust temperatures $\approx 20 \mathrm{~K}$ (e.g., Güsten et al. 1981, Molinari et al. 2011, Longmore et al. 2012). This could be explained if gas was heated by agents not affecting the dust, such as cosmic rays (see Clark et al. 2013 for recent modeling work).

The heating of CMZ gas is an unsolved problem, though. Ginsburg et al. (2016) point out that temperatures even vary within given CMZ clouds. Heating via cosmic rays, however, should provide relatively homogeneous heating throughout the clouds. Ginsburg et al. therefore conclude the gas is chiefly heated by turbulence. Immer et al. (2016) indeed find a strong correlation between gas temperature and line width supporting this picture.

\section{Conditions for Star Formation: Updates \& Personal Insights}

\subsection{High Gas Temperatures imply large Bonnor-Ebert Masses}

Here I discuss issues that are insufficiently explored elsewhere or that deserve highlighting due to new results. The former includes the application of the analysis by Ebert (1955) 
and Bonnor (1956) to the CMZ. They show that cloud fragments must exceed a mass

$$
m_{\mathrm{BE}}=20 M_{\odot} \cdot\left(T_{\text {gas }} / 50 \mathrm{~K}\right)^{3 / 2} \cdot\left(n_{\mathrm{H}_{2}} / 10^{5} \mathrm{~cm}^{-3}\right)^{-1 / 2}
$$

before they become unstable to gravitational collapse. This threshold mass is remarkably large in the Galactic Center: gas temperatures $T_{\text {gas }}$ in the CMZ exceed values found closer to Sun by a factor $\sim 5$, so that $m_{\mathrm{BE}}$ is larger by a factor $\sim 5^{3 / 2} \approx 11$ in Galactic Center clouds for fixed density $n_{\mathrm{H}_{2}}$. This suggests that star-forming cloud fragments in the CMZ are unusually massive (which might promote the formation of high-mass stars) or dense compared to regions elsewhere in the Milky Way.

\subsection{Radial Tidal Forces are not destructive}

It is often stated that only CMZ molecular clouds with densities $\gtrsim 10^{4} \mathrm{~cm}^{-3} \cdot\left(r_{\mathrm{GC}} / 75 \mathrm{pc}\right)^{-1.8}$ can survive Galactic Center tides (Güsten 1989). Current calculations, however, show that clouds at galactocentric radii of 20 to $100 \mathrm{pc}$ are generally subject to compressive tidal forces in the radial direction (e.g., Fig. 6.2 of Lucas 2015) because the gravitational force $F_{\mathrm{g}} \propto \mathrm{m} / r_{\mathrm{GC}}^{2}$ increases with increasing galactocentric radius for the observed CMZ mass profile, $m \propto r_{\mathrm{GC}}^{2}{ }^{2}$ (Launhardt et al. 2002; see Kruijssen et al. 2015 for the power-law). This updates the classical discussion by Güsten \& Downes (1980) who assume $m \propto r_{\mathrm{GC}}^{1.2}$.

\subsection{High Gas Densities from Confining Pressure}

$\mathrm{X}$-Ray images do since a long time hint at the existence of hot and tenuous CMZ gas at high pressure (Yamauchi et al. 1990; Spergel \& Blitz 1992; Muno et al. 2004). Ponti et al. (2015) suggest that candidate supernova remnants at pressure $\sim 5 \times 10^{6} \mathrm{~K} \mathrm{~cm}^{-3}$ blow shells into the gas throughout the CMZ. We may assume that CMZ molecular clouds are in balance with a lower but still similar pressure. Given gas temperatures $T_{\text {gas }} \approx 50 \mathrm{~K}$,

$$
P / k_{\mathrm{B}}=5 \times 10^{5} \mathrm{~K} \mathrm{~cm}^{-3} \cdot\left(T_{\mathrm{gas}} / 50 \mathrm{~K}\right) \cdot\left(n_{\mathrm{H}_{2}} / 10^{4} \mathrm{~cm}^{-3}\right)
$$

(where $k_{\mathrm{B}}$ is the Boltzmann constant) then implies high gas densities. This might be the chief reason why all CMZ clouds are indeed observed to have densities $n_{\mathrm{H}_{2}} \gtrsim 10^{4} \mathrm{~cm}^{-3}$.

\section{Recent Results: Star Formation in Dense Galactic Center Clouds}

\subsection{Improved Assessments of Suppressed Star Formation in Dense Gas}

Given the high gas densities of CMZ clouds, one particularly surprising feature of the region is that star formation in the dense gas of the CMZ appears to be suppressed. Taylor et al. (1993) concludes that, given massive and dense clouds, the CMZ should contain about an order of magnitude more $\mathrm{H}_{2} \mathrm{O}$ masers than observed (following Güsten \& Downes 1983 and Caswell et al. 1983; see Caswell 1996 for methanol masers). Observations with increased angular resolution eventually revealed individual clouds with little star formation (Lis et al. 1994, 2001; Lis \& Menten 1998; also see Sec. 4.2).

Our ability to quantify the relation between star formation and dense gas has improved massively over the last few years. Several studies focusing on the Solar Neighborhood (Heiderman et al. 2010; Lada et al. 2010; Evans et al. 2014) provide a framework against which Galactic Center clouds can be compared. Characterizations of the dense gas and star formation activity in the CMZ are not straightforward, as already highlighted in Sec. 2.1. The degree-scale assessments of star formation in the CMZ by Longmore et al. (2013a) build on estimates for the number of ionizing photons from WMAP data. Studies of individual clouds (e.g., Kauffmann et al. 2013b, 2016a,b) use data on HiI regions and class-II methanol masers embedded in clouds. This yields an estimate of the number 
of embedded high-mass stars, which in turn hints at the star formation rate. In future assessments of the embedded population of $\mathrm{H}_{2}$ masers provides an alternative way to gauge the star formation rate (e.g., Lu et al. 2015; also see this volume). Characterizing the dense gas is even more difficult. Following Lada et al. (2010), one may broadly consider material at $A_{V} \gtrsim 7 \mathrm{mag}$ to be "dense". Most current CMZ studies use this criterion to identify dense material on the basis of column density maps from, e.g., dust emission. Longmore et al. (2013a) also explore an approach in which all $\mathrm{NH}_{3}$-emitting gas is considered to be dense.

In summary, analysis shows that CMZ clouds are by about an order of magnitude less efficient in producing stars out of dense gas than clouds closer to Sun. This holds for averages over degree-sized parts of the region (Longmore et al. 2013a) as well as for individual clouds (Kauffmann et al. 2013b). We need to develop a detailed understanding of the conditions in the CMZ in order to understand this suppression of star formation.

\subsection{Density Structure of Molecular Clouds}

Our understanding of the internal structure of CMZ molecular clouds has increased massively over the last few years. The cloud G0.253+0.016 might serve as an example. This region was first discovered in $\mathrm{NH}_{3}$ maps of the CMZ (Güsten et al. 1981). Imaging of the CMZ in dust emission later revealed first details about the cloud structure (Lis \& Carlstrom 1994). Lis et al. (1994) realized at this point that G0.253+0.016 is very extreme in its star formation properties: the cloud concentrates a mass resembling the one of the Orion A molecular cloud in just $\sim 3 \mathrm{pc}$ radius - but there is no signifiant star formation in this object. A single faint $\mathrm{H}_{2}$ maser, such as expected in regions of low-mass star formation, is the only signpost indicating that young stars exist in this cloud. Subsequent single-dish work by Lis \& Menten (1998) and Lis et al. (2001) further refined the properties of the cloud and its star formation activity. Infrared observations of G0.253+0.016 taken during this period inspired Egan et al. (1998) and Carey et al. (1998) to coin the term "Infrared Dark Cloud" (IRDC) for regions opaque at wavelengths $\gtrsim 8 \mu \mathrm{m}$. Research on CMZ clouds then stopped for several years, given instrumental limitations. Longmore et al. (2012) revived this line of work with a fresh look at the object (now a.k.a. the "Brick") that is primarily motivated by new data from Herschel.

None of the aforementioned studies did, however, resolve the internal structure of CMZ clouds. This is a problem: single-dish data probing spatial scales $\gtrsim 1$ pc constrain how dense molecular cores capable of star formation aggregate out of the diffuse cloud medium. The observations do, however, not reveal the cores themselves on spatial scales $\lesssim 0.1$ pc where individual stars form. This means that no constraints on the immediate initial conditions for CMZ star formation can be obtained.

Interferometer observations spatially resolving CMZ clouds constitute one of the major recent advances in research exploring Galactic Center star formation. A first study of G0.253+0.016 with the Submillimeter Array (SMA) by Kauffmann et al. (2013b) reveals a puzzling trend: the cloud has a very high average density, that e.g. exceeds that of the Orion A cloud by an order of magnitude, but the cloud is essentially devoid of significant dense cores with radii $\lesssim 0.1$ pc. This trend essentially manifests in rather faint detections of $\mathrm{N}_{2} \mathrm{H}^{+}$in $S M A$ maps and an absence of significant dust continuum emission in the data. This result is not a consequence of a low sensitivity of the $S M A$ data: cloud cores resembling Orion KL but located in the CMZ, for example, would be easily detected in such data. Even more detail in G0.253+0.016 is revealed by the ALMA data of Rathborne et al. $(2014,2015)$. Their dust emission maps confirm the absence of dense cores resembling Orion KL, i.e., the relative absence of significant dense cores. Similarly, they also show that probability density functions (PDFs) of column density 
are devoid of excesses at high column density. This is typical for clouds with little star formation activity (Kainulainen et al. 2009) and quantifies that the cloud is not efficient in concentrating mass at high density.

A variety of interferometer-based studies of CMZ clouds have been published in the meantime. This includes further SMA work on G0.253+0.016 (Johnston et al. 2014), studies of Sgr C (Kendrew et al. 2013), and research into the $20 \mathrm{~km} \mathrm{~s}^{-1}$ cloud (Lu et al. 2015; also see this volume). Further work on clouds in the so-called "dust ridge" is conducted by Walker et al. (this volume; also see Walker et al. 2015). At the same time Immer et al. (2012a) used the VLA to search for faint HII regions embedded in dust ridge clouds. They find little star formation in this region, but Rodríguez \& Zapata (2013) use the same data to identify several new compact sources near and inside G0.253+0.016. Mills et al. (2015) also detect these objects, but they conclude that these are spatially extended features that are not consistent with being embedded HII regions.

The aforementioned work gives us a good idea of the spatially resolved properties in a few CMZ clouds. What is now needed is a comprehensive interferometric survey that covers most or all of the CMZ. Battersby et al. (this volume) present first results from the CMZoom project that uses the SMA to develop such an overview. In the meantime the Galactic Center Molecular Cloud Survey (GCMS; Kauffmann et al. 2016a,b) provides an SMA-based overview of the resolved properties in all major CMZ clouds (i.e., Sgr C, $20 \mathrm{~km} \mathrm{~s}^{-1}$ cloud, $50 \mathrm{~km} \mathrm{~s}^{-1}$ cloud, G0.253+0.016, and Sgr B1-off). In addition, the GCMS already includes further $A L M A$ observations of G0.253+0.016 and selected fainter CMZ clouds (Kauffmann et al., in prep.). Also, guaranteed GCMS observations mean that we will possess $A L M A$ data for all major CMZ clouds by the end of cycle 4 .

The data produce a coherent picture of CMZ cloud structure. The GCMS sample of Kauffmann et al. (2016b), e.g., shows that many CMZ clouds (i.e., $20 \mathrm{~km} \mathrm{~s}^{-1}$ cloud, $50 \mathrm{~km} \mathrm{~s}^{-1}$ cloud, G0.253+0.016, and Sgr B2) have unusually flat density profiles resembling $\varrho \propto r^{-1.3}$. Only one cloud (i.e., Sgr C - plus Sgr D, which probably resides outside the CMZ) has a steep density profile similar to a relation $\varrho \propto r^{-2}$ that would resemble the profiles expected in regions with ongoing star formation (Kauffmann et al. 2010).

In other words, CMZ star formation is at least in part suppressed because CMZ clouds are inefficient in producing high-mass dense cores of size $\lesssim 0.1$ pc that could efficiently produce stars. Section 5 discusses why CMZ clouds might have this structure.

\subsection{Kinematic Properties of Molecular Clouds}

Shetty et al. (2012) show that the velocity dispersions $\sigma(v)$ in CMZ molecular clouds exceed those prevailing elsewhere in the Milky Way by a factor $\approx 5$, when examined at a spatial scale $\sim 5$ pc. This trend has been known since the beginning of CMZ research (e.g., see Spergel \& Blitz 1992 for an early compilation). This research did, however, not constrain the spatially resolved velocity field within clouds. This is unfortunate since gas kinematics control the cloud stability against self-gravity via the virial parameter

$$
\alpha=5 \sigma^{2}(v) R /(G M)
$$

(Bertoldi \& McKee 1992; $G$ is the Gravitational constant, while $M$ and $R$ are the cloud fragment's mass and radius). The new interferometer data add this critical information.

Interestingly, data on the comprehensive GCMS sample from Kauffmann et al. (2016a) confirm that CMZ clouds have unusually high velocity dispersions when analyzed on spatial scales $\gtrsim 1 \mathrm{pc}$ - but this excess appears to vanish on smaller spatial scales. This trend had previously been found in the SMA (Kauffmann et al. 2013b) and higher-quality ALMA data (Rathborne et al. 2015) for G0.253+0.016. In other words, the interferometer 
data now indicate that relatively narrow line widths $\leqslant 1 \mathrm{~km} \mathrm{~s}^{-1}$ are found in dense cores throughout the CMZ. This implies an unusually steep CMZ linewidth-size relation.

Combination with the observed density structure indicates that CMZ clouds are gravitationally unbound on scales of a few parsec (i.e., $\alpha \gg 2$ : see Kauffmann et al. 2013a), but that substructure on smaller spatial scales is bound and potentially subject to gravitational collapse (Kauffmann et al. 2016b).

\section{Synthesis: Star Formation Ability of Galactic Center Clouds}

The discussion above can be summarized in three points. (i) The star formation ability in the dense gas of CMZ clouds is suppressed by a factor $\sim 10$, compared to regions closer to Sun. (ii) High-mass dense cores capable of significant star formation are relatively absent in CMZ clouds. ( iii) The steep linewidth-size relation prevailing in the Galactic Center might mean that bound dense cores of size $\lesssim 0.1 \mathrm{pc}$ are embedded in unbound clouds dominated by highly supersonic gas motions. The latter two factors are likely to influence star formation.

First, Kruijssen et al. (2014) argue (building on Krumholz \& McKee 2005 and Padoan \& Nordlund 2011) that the supersonic gas motions prevailing in the CMZ massively increase the threshold density required for star formation. Analysis in Kauffmann et al. (2016b) indeed gives threshold densities for star formation of $10^{7}$ to $8 \mathrm{~cm}^{-3}$ in the CMZ, where Kruijssen et al. (2014) obtain densities $\sim 10^{4} \mathrm{~cm}^{-3}$ for the Solar Neighborhood. This strongly suggests that supersonic turbulence is one of the factors suppressing CMZ star formation. Also see Bertram et al. (2015) on this point.

Note, however, that CMZ molecular clouds would actually exceed this threshold if they had a density structure typical for regions elsewhere in the Milky Way (i.e., resembling $\left.\varrho \propto r^{-2}\right)$. This is, e.g., shown in Fig. 5 of Kauffmann et al. (2016b). For this reason it seems plausible to assume that the flat density structure of clouds is the chief factor suppressing CMZ star formation: stars simply do not form because high-mass dense cores capable of significant star formation do not exist.

This, in turn, raises the question why such cores cannot form efficiently in the CMZ, i.e., why the density structure of CMZ clouds is unusually flat. We may speculate that shallow density gradients can emerge when the clouds are not tightly bound by selfgravity, so that gravity is not effective in building massive cores. The high line widths found in the CMZ would explain the low levels of gravitational binding.

The high gas velocity dispersions, finally, might be a consequence of the cloud-cloud collisions mentioned in Sec. 2.2. These could be related to the potentially self-intersecting cloud orbits proposed by Kruijssen et al. (2015; also see Lucas 2015). Also see Kruijssen et al. (this volume) and Krumholz \& Kruijssen (2015) on orbit dynamics.

Naturally, the strong magnetic field might also play a role in suppressing CMZ star formation. Observations of dust emission suggest the presence of fields with a strength $\approx 5 \mathrm{mG}$ that cloud balance against self-gravity (Pillai et al. 2015).

\section{Galactic Center Star Formation: A Template for Starbursts?}

It is often said that CMZ might serve as a template for unresolved processes that are active in nearby and more distant starburst galaxies. For example, NGC 253 (Sakamoto et al. 2011) and the Antennae Galaxies (NGC 4038/39; Wei et al. 2012) contain molecular cloud complexes with mean $\mathrm{H}_{2}$ column densities $\sim 10^{23} \mathrm{~cm}^{-2}$ : these regions must be composed of clouds with column densities of the same order, i.e., clouds resembling those 
in the CMZ. See Kruijssen \& Longmore (2013) for a related comparison between the Milky Way and other galaxies.

Still, extreme caution is required when using the CMZ as a template for the interpretation of other galaxies. The CMZ molecular clouds are, for example, apparently subject to high gas temperatures, cloud-cloud collisions, and orbital periods of just a few $10^{6} \mathrm{yr}$. It is not clear that the evolution of these clouds will resemble those of regions in starbursts that are also warm but that reside on orbits with radii of a few $10^{3} \mathrm{pc}$ and perturbation time scales $\gg 10^{6} \mathrm{yr}$. We need to disentangle all relevant processes before we can use CMZ clouds as templates to interpret the cosmos. That said, it is certainly instructive to see suppressed star formation in the CMZ. This clearly tells us that density is not the only factor controlling star formation.

\section{References}

Ao, Y., Henkel, C., Menten, K. M., et al. 2013, A\&A, 550, A135

Bally, J., Stark, A. A., Wilson, R. W., \& Henkel, C. 1988, The Astrophysical Journal, 324, 223

Bertoldi, F. \& McKee, C. F. 1992, ApJ, 395, 140

Bertram, E., Glover, S. C. O., Clark, P. C., \& Klessen, R. S. 2015, Monthly Notices of the Royal Astronomical Society, 451, 3679

Binney, J., Gerhard, O. E., Stark, A. A., Bally, J., \& Uchida, K. I. 1991, Monthly Notices of the Royal Astronomical Society, 252, 210

Bonnor, W. 1956, MNRAS, 116, 351

Carey, S. J., Clark, F. O., Egan, M. P., et al. 1998, ApJ, 508, 721

Caswell, J. L. 1996, Monthly Notices of the Royal Astronomical Society, 283

Caswell, J. L., Batchelor, R. A., Forster, J. R., \& Wellington, K. J. 1983, Australian Journal of Physics (ISSN 0004-9506), 36, 401

Chuss, D. T., Davidson, J. A., Dotson, J. L., et al. 2003, The Astrophysical Journal, 599, 1116

Clark, P. C., Glover, S. C. O., Ragan, S. E., Shetty, R., \& Klessen, R. S. 2013, The Astrophysical Journal, 768, L34

Contopoulos, G. \& Mertzanides, C. 1977, Astronomy \&3 Astrophysics, 61, 477

Cotera, A. S., Erickson, E. F., Allen, D. A., et al. 1994, in The Nuclei of Normal Galaxies: Lessons from the Galactic Center, ed. R. Genzel \& A. Harris (Dordrecht: Kluwer Academic Publishers), 217

Dahmen, G., Huttemeister, S., Wilson, T. L., \& Mauersberger, R. 1998, Astronomy and Astrophysics

Diehl, R., Halloin, H., Kretschmer, K., et al. 2006, Nature, 439, 45

Dong, H., Wang, Q. D., Cotera, A., et al. 2011, Monthly Notices of the Royal Astronomical Society, 417, 114

Dong, H., Wang, Q. D., \& Morris, M. R. 2012, Monthly Notices of the Royal Astronomical Society, 425, 884

Dunham, M., Crapsi, A., Evans II, N., et al. 2008, ApJS, 179, 249

Ebert, R. 1955, Zeitschrift für Astrophysik, 37, 217

Egan, M. P., Shipman, R. F., Price, S. D., et al. 1998, ApJ, 494, L199

Evans, N., Harvey P. M., Dunham, M., et al. 2007, Spitzer Science Center Document

Evans, N. J., Heiderman, A., \& Vutisalchavakul, N. 2014, The Astrophysical Journal, 782, 114

Figer, D. F. 2004, in The Formation and Evolution of Massive Young Star Clusters, ed. H. Lamers, L. Smith, \& A. Nota, Vol. 322 (San Francisco: Astronomical Society of the Pacific), 49

Gaume, R. A., Claussen, M. J., de Pree, C. G., Goss, W. M., \& Mehringer, D. M. 1995, ApJ, 449,663

Ginsburg, A., Henkel, C., Ao, Y., et al. 2016, Astronomy $\&$ Astrophysics, 586, A50

Güsten, R. 1989, in The Center of the Galaxy, ed. M. Morris (Dordrecht: Kluwer Academic Publishers)

Güsten, R. \& Downes, D. 1980, Astronomy and Astrophysics, 87, 6 
-. 1983, Astronomy and Astrophysics, 117, 343

Güsten, R., Walmsley, C. M., \& Pauls, T. 1981, A\&A, 103, 197

Gutermuth, R. A. \& Heyer, M. 2015, The Astronomical Journal, 149, 64

Habibi, M., Stolte, A., \& Harfst, S. 2014, Astronomy \& Astrophysics, 566, A6

Heiderman, A., Evans, N. J., Allen, L. E., Huard, T., \& Heyer, M. 2010, ApJ, 723, 1019

Henshaw, J. D., Longmore, S. N., Kruijssen, J. M. D., et al. 2016, Monthly Notices of the Royal Astronomical Society, 457, 2675

Heyer, M. \& Dame, T. 2015, Annual Review of Astronomy and Astrophysics, 53, 583

Hüttemeister, S., Dahmen, G., Mauersberger, R., et al. 1998, A\&A, 334, 646

Hüttemeister, S., Wilson, T. L., Bania, T. M., \& Martin-Pintado, J. 1993, A\&A, 280, 255

Immer, K., Kauffmann, J., Pillai, T., Ginsburg, A., \& Menten, K. M. 2016, eprint arXiv: 1607.03535

Immer, K., Menten, K. M., Schuller, F., \& Lis, D. C. 2012a, A\&A, 548, A120

Immer, K., Schuller, F., Omont, A., \& Menten, K. M. 2012b, A\&A, 537, A121

Johnston, K. G., Beuther, H., Linz, H., et al. 2014, Astronomy \& Astrophysics, 568, A56

Kainulainen, J., Beuther, H., Henning, T., \& Plume, R. 2009, A\&A, 508, L35

Kauffmann, J., Pillai, T., \& Goldsmith, P. F. 2013a, The Astrophysical Journal, 779, 185

Kauffmann, J., Pillai, T., Shetty, R., Myers, P. C., \& Goodman, A. A. 2010, ApJ, 716, 433

Kauffmann, J., Pillai, T., \& Zhang, Q. 2013b, ApJ, 765, L35

Kauffmann, J., Pillai, T., Zhang, Q., et al. 2016a, accepted to Astronomy \& Astrophysics

-. 2016b, accepted to Astronomy \& Astrophysics

Kendrew, S., Ginsburg, A., Johnston, K., et al. 2013, The Astrophysical Journal, 775, L50

Koepferl, C. M., Robitaille, T. P., Morales, E. F. E., \& Johnston, K. G. 2015, The Astrophysical Journal, 799, 53

Kruijssen, J. M. D., Dale, J. E., \& Longmore, S. N. 2015, Monthly Notices of the Royal Astronomical Society, 447, 1059

Kruijssen, J. M. D. \& Longmore, S. N. 2013, Monthly Notices of the Royal Astronomical Society, 435,2598

Kruijssen, J. M. D., Longmore, S. N., Elmegreen, B. G., et al. 2014, Monthly Notices of the Royal Astronomical Society, 440, 3370

Krumholz, M. R. \& Kruijssen, J. M. D. 2015, Monthly Notices of the Royal Astronomical Society, 453,739

Krumholz, M. R. \& McKee, C. F. 2005, The Astrophysical Journal, 630, 250

Lada, C. J., Lombardi, M., \& Alves, J. F. 2010, ApJ, 724, 687

Launhardt, R., Zylka, R., \& Mezger, P. G. 2002, Astronomy and Astrophysics, 384, 112

Lee, E. J., Murray, N., \& Rahman, M. 2012, The Astrophysical Journal, 752, 146

Lis, D. C. \& Carlstrom, J. E. 1994, ApJ, 424, 189

Lis, D. C. \& Menten, K. M. 1998, ApJ, 507, 794

Lis, D. C., Menten, K. M., Serabyn, E., \& Zylka, R. 1994, ApJ, 423, L39

Lis, D. C., Schilke, P., Bergin, E. A., et al. 2014, The Astrophysical Journal, 785, 135

Lis, D. C., Serabyn, E., Zylka, R., \& Li, Y. 2001, ApJ, 550, 761

Longmore, S. N., Bally, J., Testi, L., et al. 2013a, MNRAS, 429, 987

Longmore, S. N., Kruijssen, J. M. D., Bally, J., et al. 2013b, Monthly Notices of the Royal Astronomical Society: Letters, 433, L15

Longmore, S. N., Rathborne, J., Bastian, N., et al. 2012, ApJ, 746, 117

Lu, X., Zhang, Q., Kauffmann, J., et al. 2015, The Astrophysical Journal, 814, L18

Lucas, W. E. 2015, PhD thesis, University of St. Andrews

Martín-Pintado, J., de Vicente, P., Fuente, A., \& Planesas, P. 1997, The Astrophysical Journal, $482, \mathrm{~L} 45$

Menten, K. M., Wilson, R. W., Leurini, S., \& Schilke, P. 2009, ApJ, 692, 47

Mezger, P. G. \& Pauls, T. 1979, in The large-scale characteristics of the galaxy, Vol. 84 (Dordrecht: D. Reidel Publishing Co.), 357-365

Mills, E. A. C., Butterfield, N., Ludovici, D. A., et al. 2015, The Astrophysical Journal, 805, 72

Mills, E. A. C. \& Morris, M. R. 2013, ApJ, 772, 105 
Misiriotis, A., Xilouris, E. M., Papamastorakis, J., Boumis, P., \& Goudis, C. D. 2006, Astronomy and Astrophysics, 459, 113

Molinari, S., Bally, J., Noriega-Crespo, A., et al. 2011, ApJ, 735, L33

Morris, M. \& Serabyn, E. 1996, ARA\&A, 34, 645

Muno, M. P., Baganoff, F. K., Bautz, M. W., et al. 2004, The Astrophysical Journal, 613, 326

Nagata, T., Woodward, C. E., Shure, M., \& Kobayashi, N. 1995, The Astronomical Journal, 109,1676

Nagata, T., Woodward, C. E., Shure, M., Pipher, J. L., \& Okuda, H. 1990, The Astrophysical Journal, 351, 83

Novak, G., Chuss, D. T., Renbarger, T., et al. 2003, The Astrophysical Journal, 583, L83

Ott, J., Weiß, A., Staveley-Smith, L., Henkel, C., \& Meier, D. S. 2014, The Astrophysical Journal, 785,55

Padoan, P. \& Nordlund, A. 2011, The Astrophysical Journal, 730, 40

Pillai, T., Kauffmann, J., Tan, J. C., et al. 2015, The Astrophysical Journal, 799, 74

Ponti, G., Morris, M. R., Terrier, R., et al. 2015, Monthly Notices of the Royal Astronomical Society, 453,172

Portegies Zwart, S. F., McMillan, S. L., \& Gieles, M. 2010, ARA\&A, 48, 431

Rathborne, J. M., Longmore, S. N., Jackson, J. M., et al. 2014, The Astrophysical Journal, 795, L25

-. 2015, The Astrophysical Journal, 802, 125

Reid, M. J., Menten, K. M., Brunthaler, A., et al. 2014, The Astrophysical Journal, 783, 130

Requena-Torres, M. A., Martin-Pintado, J., Martin, S., \& Morris, M. R. 2008, The Astrophysical Journal, 672, 352

Requena-Torres, M. A., Martín-Pintado, J., Rodríguez-Franco, A., et al. 2006, Astronomy \& Astrophysics, 455, 971

Rieke, G. H. \& Lebofsky, M. J. 1985, The Astrophysical Journal, 288, 618

Riquelme, D., Amo-Baladrón, M. A., Martín-Pintado, J., et al. 2010a, Astronomy \& Astrophysics, 523, A51

-. 2012, Astronomy \& Astrophysics, 549, A36

Riquelme, D., Bronfman, L., Mauersberger, R., May, J., \& Wilson, T. L. 2010b, Astronomy \& Astrophysics, 523, A45

Rodríguez, L. F. \& Zapata, L. A. 2013, The Astrophysical Journal, 767, L13

Sakamoto, K., Mao, R.-Q., Matsushita, S., et al. 2011, ApJ, 735, 19

Schödel, R., Najarro, F., Muzic, K., \& Eckart, A. 2010, Astronomy \& Astrophysics, 511, A18

Shetty, R., Beaumont, C. N., Burton, M. G., Kelly, B. C., \& Klessen, R. S. 2012, Monthly Notices of the Royal Astronomical Society, 425, 720

Spergel, D. N. \& Blitz, L. 1992, Nature, 357, 665

Taylor, G. B., Morris, M., \& Schulman, E. 1993, The Astronomical Journal, 106, 1978

Tsuboi, M., Handa, T., \& Ukita, N. 1999, The Astrophysical Journal Supplement Series, 120, 1

Uchida, Y., Shibata, K., \& Sofue, Y. 1985, Nature, 317, 699

Walker, D. L., Longmore, S. N., Bastian, N., et al. 2015, Monthly Notices of the Royal Astronomical Society, 449, 715

Wang, Q. D., Dong, H., Cotera, A., et al. 2010, Monthly Notices of the Royal Astronomical Society, 402, 895

Wei, L. H., Keto, E., \& Ho, L. C. 2012, ApJ, 750, 136

Yamauchi, S., Kawada, M., Koyama, K., Kunieda, H., \& Tawara, Y. 1990, The Astrophysical Journal, 365, 532

Yusef-Zadeh, F., Hewitt, J. W., Arendt, R. G., et al. 2009, The Astrophysical Journal, 702, 178

Yusef-Zadeh, F., Morris, M., \& Chance, D. 1984, Nature, 310, 557 